\title{
In vitro detection of common rhinosinusitis bacteria by the eNose utilising differential mobility spectrometry
}

\author{
${ }^{1}$ Jussi Virtanen MD, ${ }^{2}$ Lauri Hokkinen BSc, ${ }^{3}$ Markus Karjalainen MSc, ${ }^{3}$ Anton Kontunen \\ MSc, ${ }^{4}$ Risto Vuento MD, PhD, ${ }^{1}$ Jura Numminen MD, PhD, ${ }^{1}$ Markus Rautiainen MD, PhD, \\ ${ }^{5}$ Niku Oksala MD, PhD, ${ }^{6 *}$ Antti Roine MD, PhD, ${ }^{1 *}$ Ilkka Kivekäs MD, PhD \\ ${ }^{1}$ Department of Otorhinolaryngology, Faculty of Medicine and Life Sciences, University of \\ Tampere and Tampere University Hospital, Tampere, Finland, ${ }^{2}$ Faculty of Medicine and Life \\ Sciences, University of Tampere, ${ }^{3}$ BioMediTech Institute and Faculty of Biomedical Sciences and \\ Engineering, Tampere University of Technology, Tampere, Finland, ${ }^{4}$ Department of Microbiology, \\ Fimlab Laboratories Ltd, Tampere, Finland, ${ }^{5}$ Department of Surgery, Faculty of Medicine and Life \\ Sciences, University of Tampere and Tampere University Hospital, Finland ${ }^{6}$ Department of \\ Surgery, Hatanpää Hospital and University of Tampere, Tampere, Finland
}

*share senior authorship

\section{Acknowledgements}

\section{Compliance with Ethical Standards}

Conflicts of Interest

Niku Oksala, Markus Karjalainen, Anton Kontunen, and Antti Roine are shareholders of Olfactomics Ltd, which is about to commercialise proprietary technology for the detection of diseases by ion mobility spectrometry.

The rest of the authors declare that they have no conflict of interest.

Ethical approval: This article does not contain any studies with human participants or animals performed by any of the authors.

Corresponding author: Jussi Virtanen, MD

Department of Otorhinolaryngology, Tampere University Hospital, PL 2000, 33521 Tampere, Finland. Tel.: +358 40 727586; fax: +358 32474366

jussi.virtanen@pshp.fi

ORCID ID: 0000-0003-0853-216X 


\begin{abstract}
Acute rhinosinusitis (ARS) is a sudden, symptomatic inflammation of the nasal and paranasal mucosa. It is usually caused by respiratory virus infection, but bacteria complicate for a small number of ARS patients. The differential diagnostics between viral and bacterial pathogens is difficult and currently no rapid methodology exists, so antibiotics are overprescribed. The electronic nose (eNose) has shown the ability to detect diseases from gas mixtures. Differential mobility spectrometry (DMS) is a next-generation device that can separate ions based on their different mobility in high and low electric fields. Five common rhinosinusitis bacteria (S. pneumoniae, $H$. influenzae, M. catarrhalis, S. aureus, and P. aeruginosa) were analysed in vitro with DMS. Classification was done using linear discriminant analysis (LDA) and k-nearest neighbour (KNN). The results were validated using leave-one-out cross-validation and separate train and test sets. With the latter, $77 \%$ of the bacteria were classified correctly with LDA. The comparative figure with $\mathrm{KNN}$ was $79 \%$. In one train-test set, $P$. aeruginosa was excluded and the four most common acute rhinosinusitis bacteria were analysed with LDA and $\mathrm{KNN}$; the correct classification rate was $83 \%$ and $85 \%$, respectively. DMS has shown its potential in detecting rhinosinusitis bacteria in vitro. The applicability of DMS needs to be studied with rhinosinusitis patients.
\end{abstract}

\title{
Keywords
}

Electronic nose, eNose, differential mobility spectrometry, acute rhinosinusitis 


\section{Introduction}

Acute rhinosinusitis (ARS) is a sudden, symptomatic inflammation of the nasal and paranasal mucosa. It is usually caused by respiratory virus infection, but bacterial prevalence remains poorly defined. In two often-cited studies [1, 2], the bacterial prevalence was $0.5-2 \%$, but according to a recent meta-analysis [3], the prevalence is likely higher. Autio et al. (2015) have found bacterial causes in $40 \%$ of cases [4]. In Finland (2016), 1.3\% of all patient visits to daily outpatient practices are due to ARS [5]. In a study conducted in Asia, it was estimated that 6-10\% of appointments with a doctor are due to ARS [6].

Antibiotics are usually prescribed, even though their effect is limited in clinically diagnosed, uncomplicated ARS [7]. The most common bacteria in ARS are Streptococcus pneumoniae, Haemophilus influenzae, Moraxella catarrhalis, and Staphylococcus aureus [8]. One explanation for the poor antibiotic response is that only $40 \%$ of ARS are bacterial culture-positive [4]. Nasal endoscopy provides the possibility to take microbiological samples from the middle meatus, but equipment is only available to ear, nose, and throat (ENT) specialists. A maxillary sinus puncture and aspiration can be considered the gold standard in diagnosing bacterial ARS, but it seems that endoscopically directed middle meatal cultures correlate well with aspiration [9].

Since the early days of medicine, doctors have linked certain diseases to specific smells. During the last decades dogs' ability to detect certain diseases by smelling have been studied [10]. Dogs use in clinical practice has pitfalls due to extensive training and their limited life-span. The odour emitted from our bodies consists of volatile organic compounds (VOCs) [11-13]. Endogenous VOCs are released as by-products of normal cell metabolism, but also directly by microbes or due to an inflammatory response to infection [11]. Certain VOCs can be specific biomarkers of diseases [13]. Most commonly, however, patterns of multiple VOCs perform better than isolated biomarkers [14]. The electronic nose (eNose) is a device that attempts to mimic mammalian olfactory sense. It comprises an array of nonselective chemical sensors, pre-processing electronics, and a computer that interprets sensor signals [15]. Different kinds of sensor arrays exist - for example, metal-oxidesemiconducting field-effect transistors (MOSFET), conducting polymer sensors, and optical sensors [15]. The eNose can also be constructed with more than one type of sensor. VOCs interact with sensors and produce a so-called smell print. The chemical characteristics of VOCs determine the response pattern of the eNose, which can be measured. The eNose does not differentiate specific components of the smell print [16], and it therefore performs a qualitative rather than a quantitative analysis. 
Differential mobility spectrometry (DMS), although not an eNose in the strictest sense, provides analogous information from gas mixtures. The DMS is highly more sensitive and selective device compared to the classic eNose. Devices with voltage adjustable ion filters have an enhanced ability to analyse complex samples without precipitation. The principle of DMS is explained thoroughly in other publications $[17,18]$. Analogously to commonly used electrophoresis, the device separates the molecular mixture to its components, creating a data matrix also known as a dispersion plot or a "chemical fingerprint" of the sample. The dispersion plot is usually represented as a heat map colorscheme. The working principle of the method is illustrated in Figure 1.

Several studies have shown the ability of the eNose to detect diseases from gaseous headspace - for example, differentiating prostatic cancer from benign prostatic hyperplasia [19] or diagnosing head and neck cancer [20], tuberculosis [21], and ventilator acquired pneumonia [22]. In a study by Thaler and Hanson (2006), the eNose was able to diagnose acute bacterial rhinosinusitis with a $72 \%$ probability [23].

There is a need for a new, easy-to-use tool for diagnosing rhinosinusitis bacteria, and the eNose has strong potential in this application. In addition, DMS provides next-generation technology to the eNose field. Our aim was to study whether DMS could identify common acute (S. pneumoniae, $H$. influenzae, M. catarrhalis) and chronic (S. aureus, P. aeruginosa) rhinosinusitis bacteria in vitro.

\section{Materials and methods}

FIMLAB (Tampere, Finland) laboratories provided the cultures of all five bacteria species. The bacteria were identified using standard biochemical methods and matrix-assisted laser desorption/ionisation time-of-flight (VITEK® MS, bioMérieux, Marcy-l'Étoile, France). Since $H$. influenzae grows only on chocolate agar, it was used as the growth medium for all the bacterial species to avoid a confounding effect from the medium.

The device, Figure 2, used in this study was an Envi-AMC (Environics Ltd, Mikkeli, Finland). It is based on DMS technology, utilising Americium ${ }^{241} \mathrm{Am}$ as an ionisation source. Dried and activated charcoal- and molecular sieve ( $5 \AA$ )-filtered compressed air was used as a carrier gas. The device was connected to a cloud database (Olfactomics Ltd, Tampere, Finland) for the monitoring and logging of the data. Two individual devices were used in the study due to the sensor malfunction of the first device. 
The measurement sessions were initiated with a baseline measurement with tap water. Each bacterial plate was measured twice, producing two measurements per sample. The device must be rinsed with water after every culture plate to control for carry-over. Based on our previous studies with the device, two water measurements are usually enough for rinsing. Each measurement produces a dispersion plot which is presented in the graphical user interface of the device. The dispersion plot was visually assessed for contamination. If significant contamination was noted, water measurements were repeated to rinse the system with water vapours until the dispersion plot had returned to baseline. The measurement cycle with two water measurements takes approximately 15 minutes. The bacteria were measured in random order with each bacteria spread over multiple sessions in order to avoid bias from sensor drift.

The analysis was made with Matlab (The MathWorks, Natick, MA, USA). Linear discriminant analysis (LDA) and k-nearest neighbour $(\mathrm{KNN})$ methods were used to classify samples. To avoid overfitting, the results were cross-validated by using leave-one-out cross-validation (LOOCV) and separate train and test sets. In LOOCV, each sample was removed from the sample set and then classified with the data of the remaining samples. When using train and test sets, a randomly selected $30 \%$ of the samples (test set) was removed from the sample set and then classified with the data of the remaining $70 \%$ (train set). This was repeated five times because of the randomisation element, and the mean classification rate of the five drives was used in the final results.

A total of 107 samples were analysed. Of these samples, there were 24 samples of $S$. aureus, 22 samples of $P$. aeruginosa, 26 samples of $S$. pneumoniae, 21 samples of $H$. influenzae, and 14 samples of $M$. catarrhalis. A total of 222 individual measurements were made (49 of $S$. aureus, 45 of $P$. aeruginosa, 52 of $S$. pneumoniae, 46 of $H$. influenzae, and 30 of $M$. catarrhalis). One sample of $S$. aureus was measured only once and two samples were measured three times. One sample of $P$. aeruginosa was measured three times. Two samples of $H$. influenzae were measured four times, as was one sample of $M$. catarrhalis.

\section{Results}

Using LOOCV, 77\% of the bacteria were classified correctly with LDA. With KNN, when $\mathrm{k}=1$, the classifications were $84 \%$ correct. When $k$ was 2 and 3, the correct classification was $93 \%$ and $76 \%$, respectively. 
Using separate train and test sets, the mean correct classification rate with LDA was 77\%. With KNN, when $\mathrm{k}=1$, the classification rate was $79.0 \%$.

We also wanted to see how well the eNose discriminates only the ARS bacteria, so P. aeruginosa samples were excluded from the data. In this scenario, with LOOCV, LDA classified $84 \%$ of the samples correctly, while $\mathrm{KNN}$, when $\mathrm{k}=1$, classified $92 \%$ of the samples correctly. With the train and test sets, LDA had a mean classification rate of $83 \%$, and with $\mathrm{KNN}$, when $\mathrm{k}=1$, the classification rate was $85 \%$.

The performance of the KNN classifiers are presented as a so-called confusion matrix in Tables 1 and 2. Examples of the dispersion plots of the bacterial samples are shown in Figure 3.

\section{Discussion}

Our results show that DMS is able to discriminate five different rhinosinusitis bacteria and the four most common ARS bacteria with a reasonably high accuracy. Since the microbiological cause of ARS is challenging and time-consuming to diagnose, these results lay the foundations for a new diagnostic approach.

To our knowledge, there are few in vitro studies concerning the eNose and ENT bacteria [24, 25]. Lai et al. (2002) used Cyranose 320 (Cyrano Technologies, Pasadena, CA, USA) in their in vitro analysis of ten common upper respiratory bacteria, which included those tested in our study, but also others, such as Streptococcus Group A, Klebsiella species, and Proteus mirabilis. The eNose was able to distinguish three tested bacteria from control swabs. Lai et al. also tested discrimination ability across a variety of bacterial species, but in quite many cases, the eNose gave false results. Moreover, cross-validation was not used, giving overoptimistic results for the performance of the eNose [24].

Thaler et al. (2008) examined the ability of the Cyranose 320 to distinguish biofilm-producing Pseudomonas and Staphylococcus from non-biofilm-producing strains of the same species [25]. The samples were tested over 24 days along with control data saline to check the consistency of the sensor responses. Consistent responses were received for 22 days. Internal validation was performed using the leave-one-day-out method. Furthermore, they also divided the data into training and testing sets with success rates ranging from $72 \%$ to $100 \%$, which are quite similar to ours. Although Thaler et al.'s sample size was larger (198 samples of each bacteria), our study tested five different bacteria species. The technology in Cyranose 320 relies on conducting-polymer sensors, which have lower 
sensitivity, specificity, and accuracy than DMS [26]. In addition, the conducting-polymer sensors suffer from irreversible contamination.

Our analysis also used bacteria in vitro. There are a few limitations regarding the study method. First, we acquired bacterial plates from a laboratory, and there was variance in the duration of plate storage in the refrigerator before measurements. Some were measured on the same day and some after a few days. Analysing all species over multiple sessions minimised bias from this variation. It is not known if some or all of the bacteria on the plate had died before measurement. This could lead to a weaker smell print but, on the other hand, it is also possible that bacteriolysis releases molecules that actually enhance detection. In addition, there was unavoidable variance in the confluence of the bacteria on the agar plate, which could affect DMS performance. As seen from the confusion matrix presented in Table 1, the device distinguishes all $S$. aureus correctly, but there are false estimates between $P$. aerigunosa and $M$. catarrhalis and also between $H$. influenzae and $S$. pneumoniae, demonstrating that there are probably similarities in the gaseous headspace of these bacteria. We must also emphasise that the specific molecules detected by the eNose are unknown. Thus, the smell print might change when the sample is acquired from a patient because there are also other molecules affecting it, such as those produced by the host inflammatory response. Two preliminary clinical trials have been conducted concerning rhinosinusitis diagnostics with the eNose [23, 27]. Nasal out-breath was sampled in both studies. The correct results varied between $60 \%$ and $72 \%$ with a modest sample size and limited cross-validation.

There are many types of eNose sensor, and they all have their strengths and weaknesses. For example, surface acoustic wave sensors benefit from their high sensitivity to a broad spectrum of VOCs and relatively low costs in biomedical applications, but they suffer from reproducibility issues [14]. Metal oxide-semiconductor sensors, on the other hand, are easier to reproduce but suffer from poor long-term stability.

The eNose in this study is based on DMS technology [17], which has been studied in various medical applications [18], but to our knowledge there are no publications regarding bacteria affecting the ENT area. The advantages of DMS include its high sensitivity and its ability to work at room temperature. Device-to-device variation is also limited, as we demonstrated in our study by changing the sensor during the study period. Its disadvantage is its susceptibility to contamination, which requires the sensors to be rinsed with water vapours between measurements.

The eNose produces multidimensional data, which requires pre-processing and classification to build a model. A variety of techniques for data analysis exists, but there is no consensus as to which one is 
best [28]. Therefore, depending on the sensor and application, different methods should be tested. A recent review [28] aimed to give an overview of the currently used dimension reduction, classification, and validation methods. Although cross-validation is considered crucial [19], a literature review regarding the investigation of exhaled breath and eNoses yielded 46 studies, with internal validation performed in $29(63 \%)$ studies and external validation performed only in $7(15 \%)$ studies. In our study, the optimal data analysis method gave correct results in $93 \%$ of cases. To minimise the risk of overfitting, we utilised the leave-one-out method for internal validation in all measurements. External validation with the train-test set, which is considered the most robust method of cross-validation [29], yielded a correct classification rate of 79\%, which is still an excellent result considering the modest sample size. We therefore consider our methods of cross-validation adequate.

At the moment, Gram staining and a bacterial culture are the gold standard for microbial identification from samples. They are cheap and easy to do but requires laboratory setting and staff. For all the bacteria tested in our study, it usually takes one day to get the results in culture and approximately a day more for information about antibiotic sensitivity.

DMS can analyse complex samples in real-time without prior sample preparation. For example, it can differentiate Clostridium difficile-positive stool samples from negative ones with high accuracy [30]. DMS has also shown ability to differentiate methicillin-resistant $S$. aureus from methicillin-sensitive S. aureus [31]. Its high sensitivity allows detection of compounds even in low concentration. Our device is easy to use and the actual measurements can be done by almost anyone after brief instructions.

Although in this study we analysed bacterial plates, the same methodology can be applied to a clinical setting. Samples acquired from rhinosinusitis patients can be analysed with DMS easily. An empty agar plate can be covered with pus collected from the maxillary sinus or middle meatus and analysed in the same manner as we did with the bacterial plates. The results can be compared with the results presented in this study. Our device does not have data-processing software within itself. Therefore, it does not give an instant result which bacteria grows in a sample. Thus, it is not yet possible to use the device in a clinical practice. Technically, there is only need for an analysis software to be integrated to the device to have an instant result after measurement. The device has a clear potential for pointof-care use since it is relatively compact in size, works in room temperature, utilizes ambient air as carrier gas and tap water for rinsing. Since DMS analyses bacterial headspace, it is also relatively easy to develop an analytical method for the analysis of exhaled nasal air, utilising, for example, 
existing methods for nasal nitric oxide measurement, analogous to the sampling used in exhaled breath studies [32].

\section{Conclusion}

The diagnostics of bacterial ARS are uncertain, so there is a need for a new diagnostic tool. DMS has proven its potential in detecting rhinosinusitis bacteria in vitro. The positive results remained after rigorous cross-validation, indicating that the performance of DMS is realistic. Therefore, the device is most likely able to distinguish between unknown samples. More studies are needed on the clinical applications of DMS in rhinosinusitis patients.

\section{References}

1. Dingle J, Badger G \& Jordan WJ (1964) Illness in the home: a study of 25,000 illness in a group of Cleveland families. Cleveland, The Press of Western Reserve University.

2. Berg O, Carenfelt C, Rystedt G \& Änggård A (1986) Occurrence of asymptomatic sinusitis in common cold and other acute ENT-infections. Rhinology 24: 223-225.

3. Smith SS, Ference EH, Evans CT, Tan BK, Kern RC, Chandra RK (2015) The prevalence of bacterial infection in acute rhinosinusitis: a Systematic review and meta-analysis. Laryngoscope 125:57-69. https://doi.org/10.1002/lary.24709

4. Autio T, Tapiainen T, Koskenkorva T, Närkiö M, Lappalainen M, Nikkari S, Hemmilä H, Koskela KA, Koskela M, Koivunen P, Alho OP (2015) The role of microbes in the pathogenesis of acute rhinosinusitis in young adults. Laryngoscope 125:1-7. http:s//doi.org/10.1002/lary.24862

5. National Institute for Health and Welfare. Out-patient treatment notifications in primary care in 2016. https://www.thl.fi/fi/tilastot/tiedonkeruut/perusterveydenhuollon-avohoidonhoitoilmoitus-avohilmo/raportit. Accessed 18 October 2017

6. Wang D, Wardani R, Sinqh K, Thanaviratananich S, Vicente G, Xu G, Zia M, Gulati A, Fang S, Shi L, Chan Y, Price D, Lund V, Mullol J, Fokkens W (2011) A survey on the management of acute rhinosinusitis among Asian physicians. Rhinology 49:264-71. https://doi.org/10.4193/Rhino10.169

7. Lemiengre MB, van Driel ML, Merenstein D, Young J \& De Sutter AI (2012) Antibiotics for clinically diagnosed acute rhinosinusitis in adults. Cochrane Database Syst Rev 10: CD006089. https://doi.org/10.1002/14651858.CD006089.pub4

8. Payne SC, Benninger MS (2007) Staphylococcus aureus is a major pathogen in acute bacterial rhinosinusitis: a meta-analysis. Clin Infect Dis 45:121-7.

9. Benninger MS, Payne SC, Ferguson BJ, Ahmad N (2006) Endoscopically directed middle meatal cultures versus maxillary sinus taps in acute bacterial maxillary rhinosinusitis: a meta-analysis. Otolaryngol Head Neck Surg 134:3-9.

10. Bijland LR, Bomers MK, Smulders YM (2013) Smelling the diagnosis: a review on the use of scent in diagnosing disease. Netherlands J Med 71:300-7. 
11. Sethi S, Nanda R, Chakraborty T (2013) Clinical application of volatile organic compound analysis for detecting infectious diseases. Clin Microbiol Rev 26:462-75. https://doi.org/10.1128/CMR.00020-13

12. Shirasu M, Touhara K (2011) The scent of disease: volatile organic compounds of the human body related to disease and disorders. J Biochem 150:257-266. https://doi.org/10.1093/jb/mvr090

13. Wilson D (2015) Advances in Electronic-Nose Technologies for the Detection of Volatile Biomarker Metabolites in the Human Breath. Metabolites 5:140-163. https://doiorg/10.3390/metabo5010140

14. Lourenço C, Turner C (2014) Breath Analysis in Disease Diagnosis: Methodological Considerations and Applications. Metabolites 4:465-498. https://doi.org/10.3390/metabo4020465

15. Arshak K, Moore E, Lyons G.M, Harris J, Clifford, S (2004) A review of gas sensors employed in electronic nose applications. Sensor Review 24:181-198. https://doi.org/10.1108/02602280410525977

16. Wilson D, Baietto M (2011) Advances in Electronic-Nose Technologies Developed for Biomedical Applications. Sensors 11:1105-1176. https://doi.org/10.3390/s110101105

17. Kolakowski B, Mester Z (2007) Review of applications of high-field asymmetric waveform ion mobility spectrometry (FAIMS) and differential mobility spectrometry (DMS). Analyst 132:842-86. https://doi.org/10.1039/b706039d

18. Covington, JA, van der Schee MP, Edge SL, Boyle B, Savage S, Arasaradnam RP (2015) The application of FAIMS gas analysis in medical diagnostics. Analyst 140:6775-6781. https://doi.org/10.1039/c5an00868a

19. Roine A, Veskimäe E, Tuokko A, Kumpulainen P, Koskimäki J, Keinänen TA, Häkkinen MR, Vepsäläinen J, Paavonen T, Lekkala J, Lehtimäki T, Tammela TL, Oksala NK (2014) Detection of prostate cancer by an electronic nose: a proof of principle study. J Urol 192:230-4. https://doi.org/10.1016/j.juro.2014.01.113

20. Leunis N, Boumans ML, Kremer B, Din S, Stobberingh E, Kessels AG, Kross KW (2014) Application of an electronic nose in the diagnosis of head and neck cancer. Laryngoscope 124:1377-81. https://doi.org/10.1002/lary.24463

21. Kolk A, Hoelscher M, Maboko L, Jung J, Kuijper S, Cauchi M, Bessant C, van Beers S, Dutta R, Gibson T, Reither K (2010) Electronic-nose technology using sputum samples in diagnosis of patients with tuberculosis. J Clin Microbiol 48:4235-8. https://doi.org/10.1128/JCM.00569-10

22. Hockstein NG, Thaler ER, Torigian D, Miller WT Jr, Deffenderfer O, Hanson CW (2004) Diagnosis of pneumonia with an electronic nose: correlation of vapor signature with chest computed tomography scan findings. Laryngoscope 114:1701-5.

23. Thaler ER, Hanson C (2006) Use of an electronic nose to diagnose bacterial sinusitis. Am J Rhinol 20:170-2.

24. Lai SY, Deffenderfer OF, Hanson W, Phillips MP, Thaler ER (2002) Identification of upper respiratory bacterial pathogens with the electronic nose. Laryngoscope 112:975-9.

25. Thaler ER, Huang D, Giebeig L, Palmer J, Lee D, Hanson CW, Cohen N (2008) Use of an electronic nose for detection of biofilms. Am J Rhinol. 22:29-33. https://doi.org/10.2500/ajr.2008.22.3126

26. Arasaradnam RP, Covington JA, Harmston C, Nwokolos CU (2014) Review article: next generation diagnostic modalities in gastroenterology - gas phase volatile compound biomarker detection. Aliment Pharmacol Ther 39:780-789.

https://doi.org/10.1111/apt.12657 
27. Mohamed EI, Bruno E, Linder R, Alessandrini M, Di Girolamo A, Pöppl SJ, Puija A, De Lorenzo A (2003) A novel method for diagnosing chronic rhinosinusitis based on an electronic nose. An Otorrinolaringol Ibero Am 30:447-57.

28. Leopold JH, Bos LD, Sterk PJ, Schultz MJ, Fens N, Horvath I, Bikov A, Montuschi P, Di Natale C, Yates DH, Abu-Hanna A (2015) Comparison of classification methods in breath analysis by electronic nose. J Breath Res 9:046002. https://doi.org/10.1088/17527155/9/4/046002

29. Marco S (2014) The need for external validation in machine olfaction: emphasis on healthrelated applications. Anal Bioanal Chem 406:3941-3956. https://doi.org/10.1007/s00216014-7807-7

30. Bomers MK, Menke FP, Savage RS, Vandenbroucke-Grauls CM, van Agtmael MA, Covington JA, Smulders YM (2015) Rapid, accurate, and on-site detection of C. difficile in stool samples. Am J Gastroenterol 110:588-94. https://doi.org/10.1038/ajg.2015.90

31. Saviauk T, Kiiski JP, Nieminen MK, Tamminen NN, Roine AN, Kumpulainen PS, Hokkinen LJ, Karjalainen MT, Vuento RE, Aittoniemi JJ, Lehtimäki TJ, Oksala NK (2018) Electronic Nose in the Detection of Wound Infection Bacteria from Bacterial Cultures: A Proof-of-Principle Study. Eur Surg Res 59:1-11. https://doi.org/10.1159/000485461

32. Peng G, Tisch U, Adams O, Hakim M, Shehada N, Broza YY, Billan S, Abdah-Bortnyak R, Kuten A, Haick H (2009) Diagnosing lung cancer in exhaled breath using gold nanoparticles. Nat Nanotechnol 4:669-73. https://doi.org/10.1038/nnano.2009.235

\section{Legend to the Figure:}

Figure 1. The working principle of DMS. DMS is analogous to electrophoresis (A) in which molecules are driven through the medium by an electrical current. The molecules in the medium cause drag that depends on the size and shape of the molecule. The electrical charge and mass also affect 
the speed of the molecule. Due to these factors, different molecules travel at different speeds. In DMS (B) molecules that evaporate from the sample are driven with a flow of air. They are given an electric charge by ionization, allowing discrimination of molecules according to their charge. The molecules then enter separation phase where they are exposed to intermittent high- and low electric fields. Ionized molecules move towards the oppositely charged plate at different speed in low and high fields, thus resulting in another dimension for separation. Analogously to electrophoresis, the collisions with medium also discriminate the molecules according to their size and shape. After separation, molecules collide with the detector, resulting in a signal.

Figure 2. Illustration of the DMS device. The bacterial plates were placed into the measurement chamber (no 1) from which the evaporated molecules were channelled to the analyzer (no 2).

Figure 3. Examples of dispersion plots for positive ion spectrums. 
Table 1. Confusion matrix of all the bacterial samples using $\mathrm{KNN}$, when $\mathrm{k}=1$. The rows represent true bacterial samples and the columns represent the class in which the sample was classified. For example, there were 46 samples of $H$. influenzae; 43 of them were classified correctly and 3 of them were classified as $S$. pneumoniae.

\section{Estimate}

\begin{tabular}{|c|c|c|c|c|c|c|}
\hline & & $\begin{array}{c}\text { S. } \\
\text { aureus }\end{array}$ & $\begin{array}{c}P . \\
\text { aeruginosa }\end{array}$ & $\begin{array}{c}\text { S. } \\
\text { pneumoniae }\end{array}$ & $\begin{array}{c}\text { H. } \\
\text { influenzae }\end{array}$ & $\begin{array}{c}\text { M. } \\
\text { catarrhalis }\end{array}$ \\
\hline & S. aureus & 49 & 0 & 0 & 0 & 0 \\
\hline & P. aeruginosa & 1 & 33 & 0 & 1 & 10 \\
\hline \multirow[t]{3}{*}{ True } & S. pneumoniae & 2 & 0 & 43 & 5 & 2 \\
\hline & H. influenzae & 0 & 0 & 3 & 43 & 0 \\
\hline & M. catarrhalis & 0 & 8 & 3 & 0 & 19 \\
\hline
\end{tabular}


Table 2. Confusion matrix (KNN, when $\mathrm{k}=1)$ of the bacterial samples without $P$. aeruginosa. The interpretations were made in the same manner as in Table 1.

\section{Estimate}

S. aureus S. pneumoniae H. influenzae M. catarrhalis

\begin{tabular}{lcccc}
\hline S. aureus & 49 & 0 & 0 & 0 \\
S. pneumoniae & 2 & 43 & 5 & 2 \\
H. influenzae & 0 & 3 & 43 & 0 \\
M. catarrhalis & 0 & 3 & 0 & 27 \\
\hline
\end{tabular}




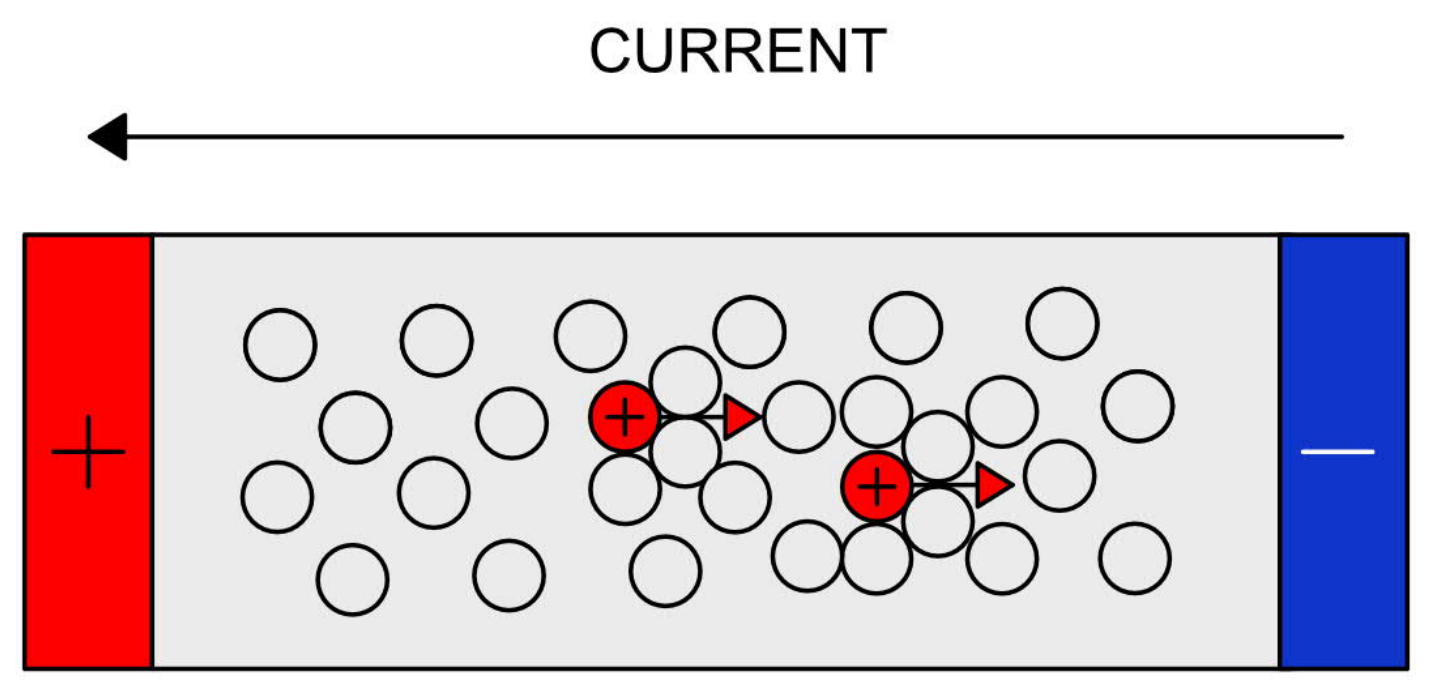

A) ELECTROPHORESIS 


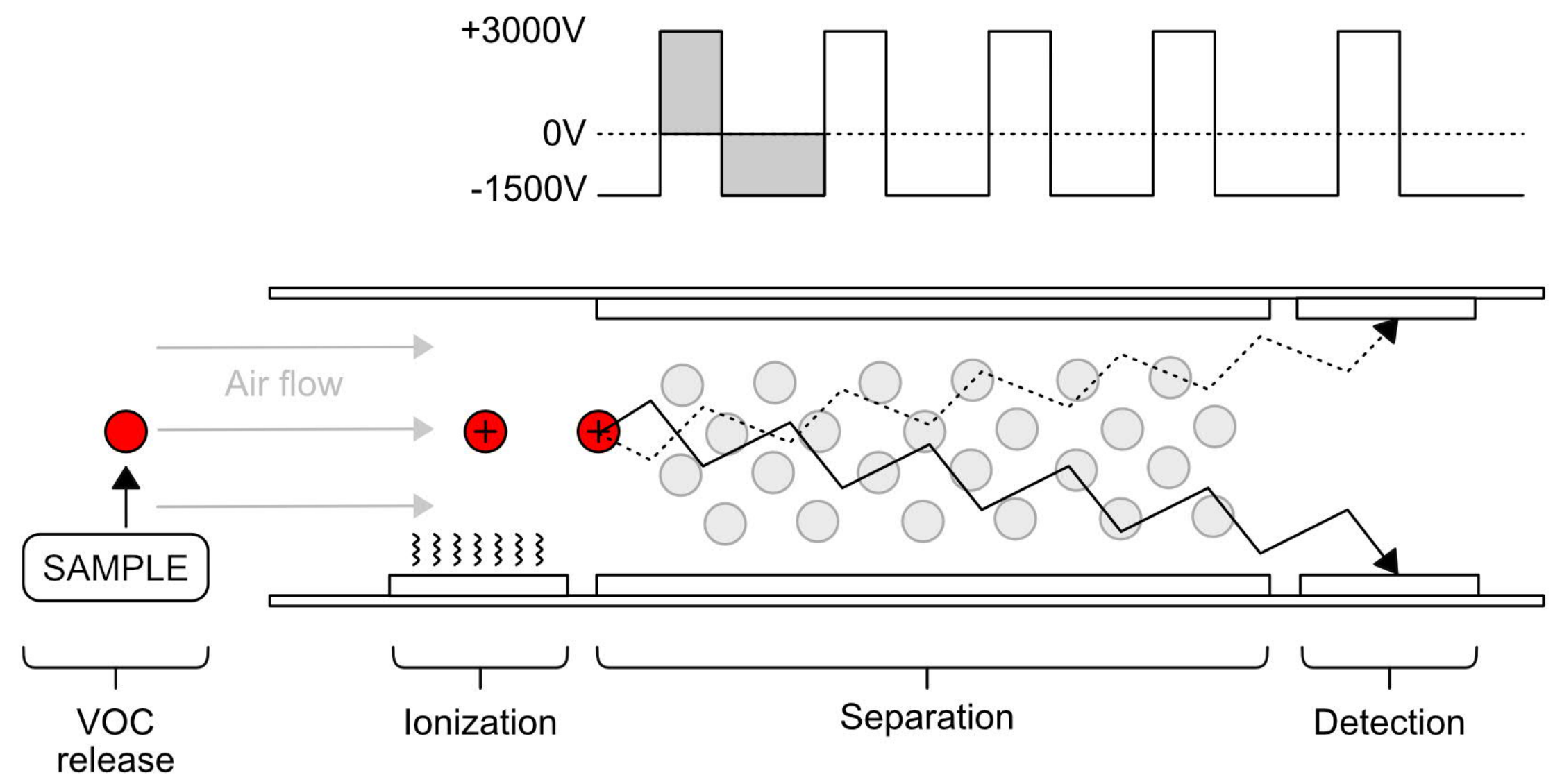

B) DIFFERENTIAL MOBILITY SPECTROMETRY 


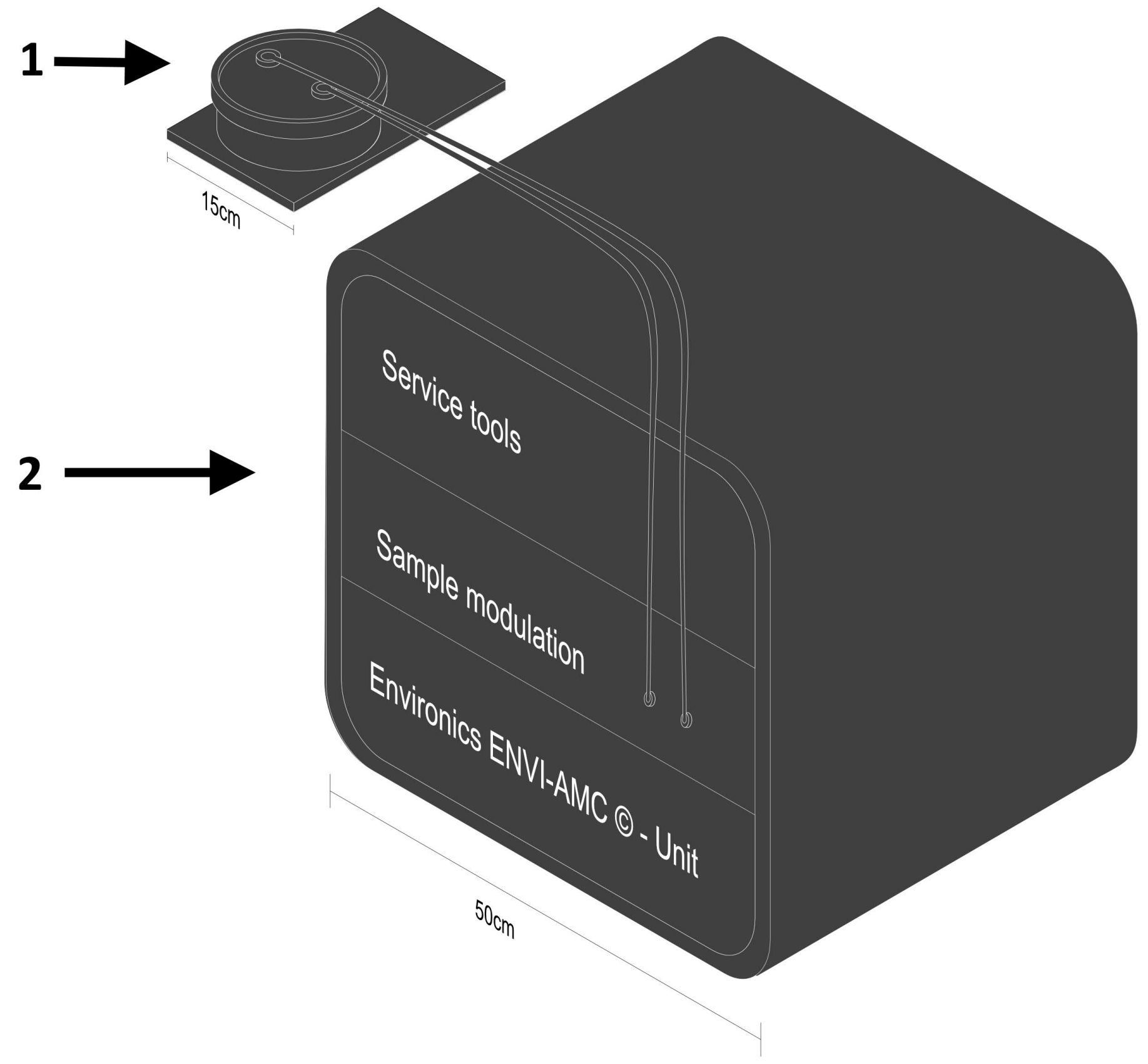




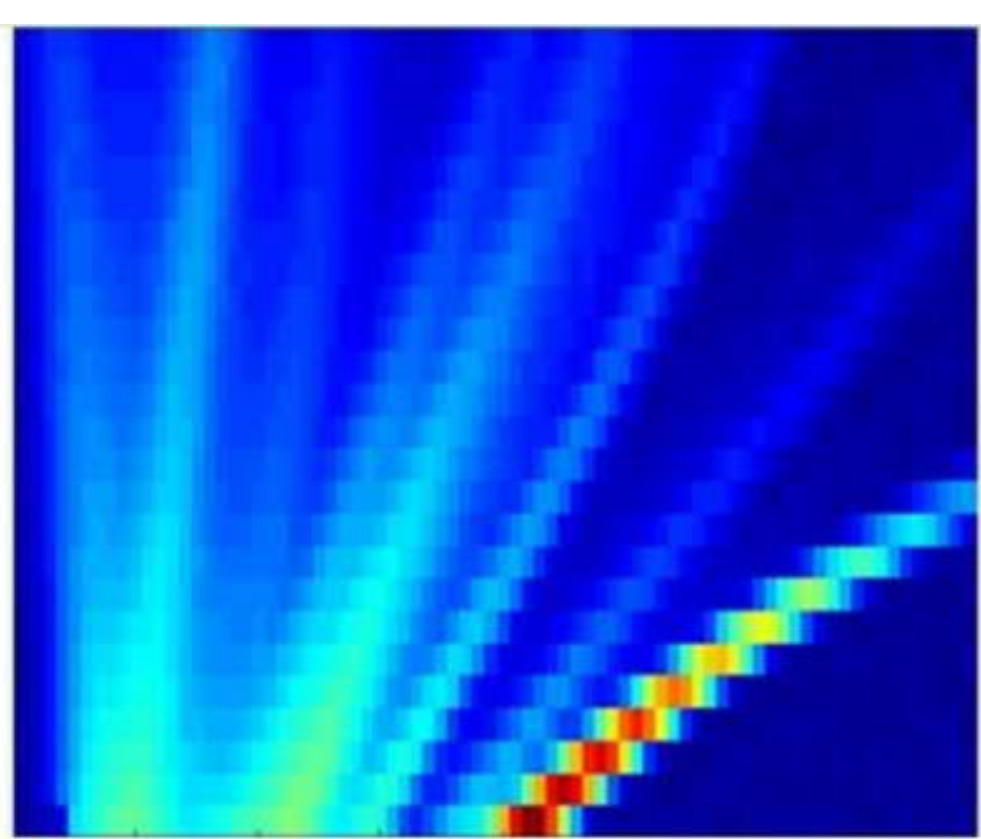

$P$. aeruginosa

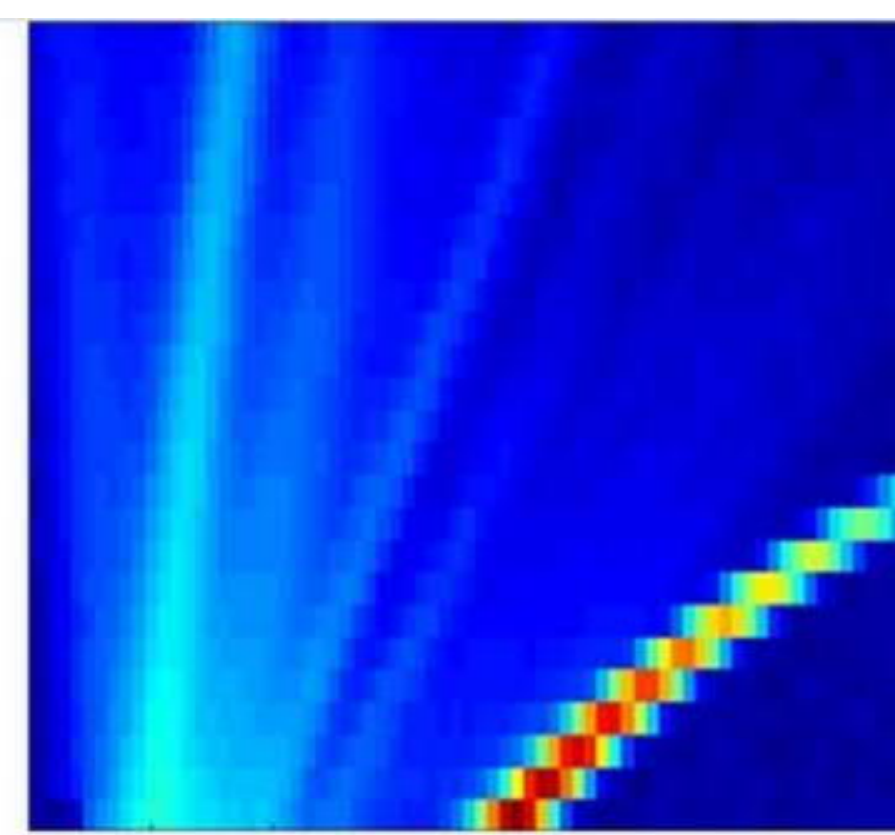

S. pneumoniae

S. aureus

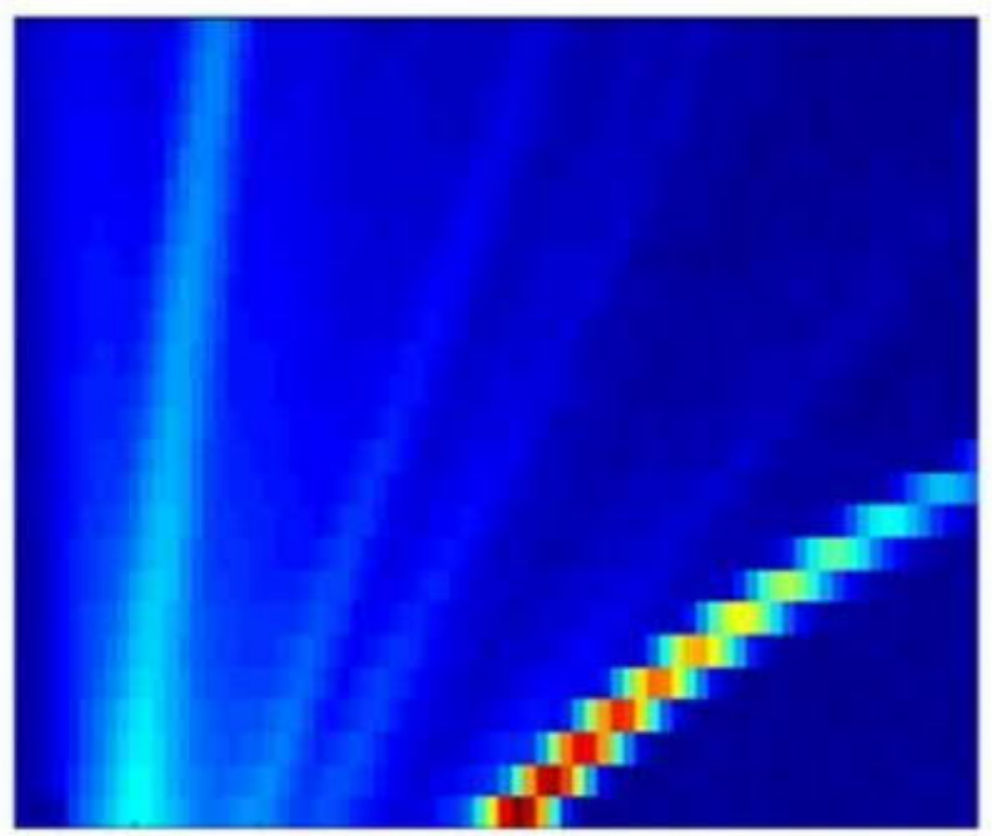

$H$. influenzae

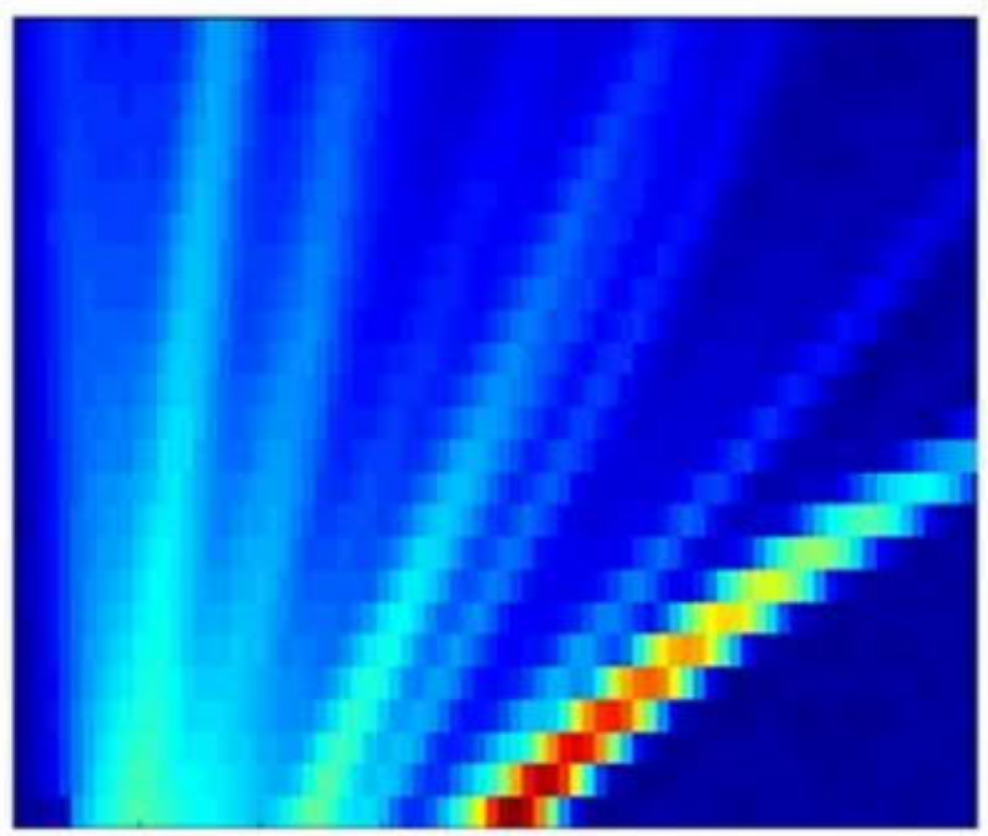

M. catarrhalis 